\title{
Determinação em Linha de Tamanho de Partículas na Polimerização em Suspensão
}

\author{
Alexandre F. Santos, Enrique L. Lima e José Carlos Pinto
}

\begin{abstract}
Resumo: A técnica de espectrofotometria na região do infra-vermelho próximo (NIR) é usada nesse trabalho para avaliar e controlar de forma contínua e em linha a evolução do tamanho médio de partícula em polimerizações de estireno em suspensão. Os resultados obtidos mostram que os espectros de NIR são sensíveis a modificações das condições de operação, podendo dessa forma prover informações sobre a evolução dinâmica da distribuição de tamanhos de partícula e sobre a estabilidade da suspensão durante a polimerização. Observa-se que a manipulação das variáveis normalmente utilizadas para modificar as curvas de distribuição de tamanhos, tais como a velocidade de agitação e a concentração de agente estabilizante, não causa modificações significativas na cinética da polimerização. Finalmente, experimentos mostram que a monitoração e controle contínuo da polimerização com a técnica de NIR permite projetar e obter distribuições de tamanho com valores médios pré-especificados, o que aponta para potencial desenvovlvimento de aplicações industriais.
\end{abstract}

Palavras-chave: Infravermelho próximo, tamanho de partícula, polimerização, suspensão, análise em linha.

\section{Introdução}

Nos processos de polimerização em suspensão, a morfologia da resina (distribuição de tamanhos de partícula (DTP), formato e porosidade) constitui importante parâmetro de qualidade a ser controlado. No caso da distribuição de tamanhos, a obtenção de resinas com tamanhos fora de especificação pode dificultar o processamento nas etapas de extrusão e transformação, acarretando sérios prejuízos à qualidade do produto final (Yuan et al., 1991). Grandes frações de partículas finas constituem um risco de poluição ambiental, implicando o uso de ciclones na etapa de purificação por ocasião da lavagem e secagem por corrente de ar (Bishop, 1971;
Grulke, 1989). Partículas muito grandes geralmente apresentam problemas de fluxo durante o processamento, não se fundindo completamente e, conseqüentemente, acarretam a formação de um defeito no produto final denominado "olho-de-peixe" (fish eye) (Sarvetnick, 1969). Tal defeito consiste na presença de nódulos que prejudicam a aparência e a durabilidade do artefato, servindo como ponto de ruptura para a peça sob tensão. Além disso, o olho-de-peixe constitui o principal local para degradação e descoloração do material (Sarvetnick, 1969; Rubin, 1972).

Muitos esforços têm sido efetivados para o desenvolvimento de técnicas de análise em linha de tamanho de partículas em meios heterogêneos. 
Contudo, a maioria dessas investigações estão relacionadas a sistemas diluídos ou em ausência de reação química (Clifford et al., 1993; Chatzi et al., 1997; LIU et al., 1997; POPP et al., 1998). A conjugação entre medida em linha de tamanho de partícula e reação química permanece ainda como um obstáculo a ser superado.

Uma técnica que vem sendo amplamente utilizada na análise de propriedades em processos químicos é a espectrofotometria na região do infravermelho próximo (NIR, near-infrared). A região NIR produz espectros de absorção complexos, desprovidos de bandas bem definidas para os diferentes grupos funcionais (Bjorsvik, 1996). As absorbâncias da região resultam de harmônicos (overtones) ou bandas de combinação das vibrações fundamentais que surgem na região do infravermelho médio (2500 nm - $16000 \mathrm{~nm}$ ), sendo tipicamente mais fracas que as transições fundamentais correspondentes. Devido a este comportamento pouco específico, reflexo da presença de picos de absorção largos e sobrepostos, a região do infravermelho próximo foi inicialmente avaliada como de difícil interpretação e suas bandas eram consideradas muito fracas para aplicações práticas. Entretanto, com o desenvolvimento de métodos multivariáveis para análise de dados, tais como análise em componentes principais (PCA) e regressão nos mínimos quadrados parciais (PLS), foi possível o crescimento exponencial do número de aplicações da espectrofotometria no infravermelho próximo na análise em linha de propriedades químicas e físicas de reatores e correntes de processo. Isto porque, diferente da metodologia tradicional utilizada nas análises na região do infravermelho médio, que envolve a busca do comprimento de onda característico do constituinte, as técnicas multivariáveis permitiram o emprego de todo o espectro para a construção de modelos de calibração confiáveis (Hall et al., 1996).

A consagração da espectrofotometria no infravermelho próximo se deu com a introdução de feixes de fibras óticas para a transmissão de luz. Isto foi possível pois o quartzo, constituinte básico da fibra ótica, possui excelente capacidade de transmissão de radiação de comprimentos de onda na região NIR (SIESLER, 1991). Desta maneira, abriu-se caminho para a construção de instrumentos remotos, onde o espectrofotômetro, munido de todas as suas partes essenciais (fonte de luz, monocromador, detetor,...), poderia ser instalado fora do ambiente de processo e a luz seria transferida do espectrofotômetro - via feixe de fibras óticas - até a amostra, sendo transferida de volta para o instrumento após transmissão ou reflexão pela amostra. Com esta nova concepção, a espectrofotometria deixa de ser uma atividade essencialmente de laboratório e passa a ingressar nas plantas industriais, porém, assumindo nova configuração do ponto de vista da instrumentação: espectrofotômetro, feixe de fibras óticas e probe este último consiste na sonda que contém o caminho ótico para iluminação das amostras. Tal configuração vem possibilitando a coleta de espectros de correntes de processos e sistemas reacionais insitu, viabilizando análises em tempo real para fins de controle.

Neste trabalho apresenta-se aplicação da técnica de espectrofotometria no infravermelho próximo no monitoramento e controle em tempo real de tamanhos de partícula, em um reator de polimerização em suspensão. Tal aplicação foi possível através da elaboração de modelos empíricos, relacionando espectro de infravermelho próximo e tamanho médio de partícula de polímero. O modelo final, obtido a partir da regressão PLS (partial least squares), foi capaz de descrever bem a evolução dos tamanhos de partícula em sistemas de polimerização de estireno em suspensão, efetuando predições dentro do intervalo de confiança da média experimental, para um grande número de experimentos e condições em tempo real, viabilizando a implementação de rotinas de controle em linha de tamanho de partículas.

\section{Experimental}

Foram realizadas algumas corridas de polimerização de estireno em suspensão com espectrofotômetro NIR em linha, utilizando peróxido de benzoíla como iniciador, em reator do tipo tanque agitado, dentro do esquema da Figura 1. O reator utilizado foi de vidro borossilicato (1 litro), encamisado, equipado com condensador de refluxo, controlador de temperatura e agitador mecânico do tipo hélice naval (FISATOM $713 \mathrm{~T}$ ) capaz de operar na faixa de 90 a $6300 \mathrm{rpm}$. Os espectros de infravermelho foram coletados a intervalos 


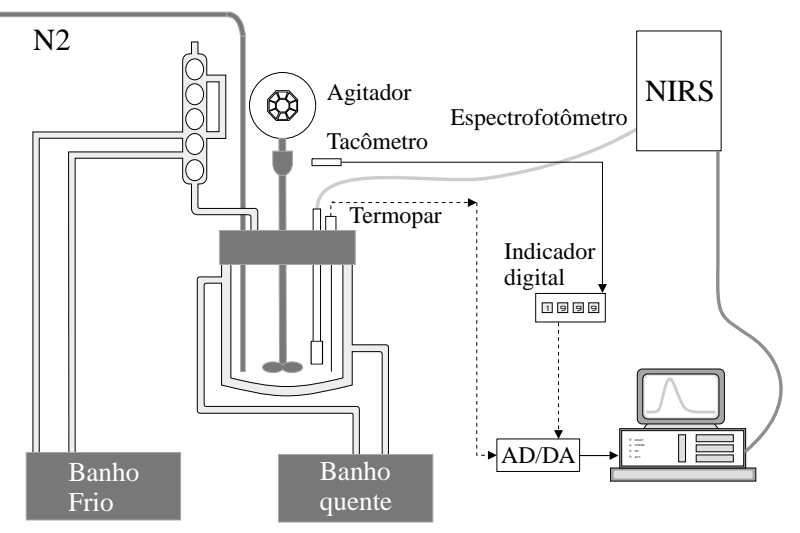

Figura 1. Esquema da unidade experimental

regulares de 3 minutos com o espectrofotômetro em linha NIRS-6500 (NIRSystems Inc.), operando no modo transmitância na região espectral de $400 \mathrm{~nm}$ a $2500 \mathrm{~nm}$. As aquisições de dados, as análises nos mínimos quadrados parciais e os tratamentos matemáticos dos espectros foram efetuados através do software Near-infrared Spectral Analysis Software (NSAS, 1993), fornecido pelo fabricante do instrumento. Ao longo dos experimentos, a velocidade de agitação foi medida com o uso de um tacômetro (Takotron TD2004-C).

Amostras do meio reacional foram coletadas em intervalos regulares para determinação de conversão, peso molecular médio e tamanho de partícula. A conversão de monômero em polímero foi determinada gravimetricamente. A determinação da distribuição de pesos moleculares se deu por cromatografia de permeação em gel. As análises de DTP foram realizadas via microscopia eletrônica de varredura (SANTOS et al., 1998).

Os reagentes empregados nos experimentos foram o estireno (Nitriflex Resinas S.A.), peróxido de benzoíla (Quimibrás), água destilada, poliestireno sulfonado (PES, Nitrilfex Resinas S.A.) e poli(álcool vinílico) com grau de hidrólise de 9999,8\% (PVA, Fine Chemicals). Visando simular condições reais de operação industrial, os reagentes foram usados conforme recebidos, sem purificação adicional.

\section{Calibração}

Para construção do modelo empírico de calibração (PLS) que relaciona tamanho médio de partícula com espectro, foi adotado o procedimento apresentado anteriormente (SANTOS et al, 1998). O conjunto de dados usado compreende os dados apresentados no trabalho anterior e dados adicionais utilizados para ampliar a faixa de validade do modelo de calibração. As condições operacionais dos experimentos adicionais são exibidas na Tabela 1. Os experimentos 1-6 e 18 foram incluídos no conjunto de dados e usados na calibração, conforme discutido abaixo. Os experimentos 17 e 19 foram aplicados apenas na validação do modelo.

$\mathrm{O}$ teste de validação cruzada ilustrado na Figura $2 \mathrm{~A}$ mostra a evolução típica do desvio padrão das predições do modelo (usando conjunto de dados de validação) e a evolução dos coefici-

Tabela 1. Condições e dados experimentais dos experimentos de calibração e validação

\begin{tabular}{|c|c|c|c|c|c|c|c|c|}
\hline Exp* & $\begin{array}{l}\text { Velocidade de } \\
\text { agitação (rpm) }\end{array}$ & $\begin{array}{c}\text { Tempo } \\
\text { (h) }\end{array}$ & $\begin{array}{c}\text { Conversão } \\
(\%)\end{array}$ & $\begin{array}{c}\mathbf{T P} \pm \sigma \\
(\mu \mathrm{m})\end{array}$ & Exp & $\begin{array}{c}\text { Velocidade de } \\
\text { agitação (rpm) }\end{array}$ & $\begin{array}{l}\text { Tempo } \\
\text { (h) }\end{array}$ & {$[\mathrm{PES}], \mathrm{g} / \mathrm{L}$} \\
\hline 1 & 1960 & 3,33 & 70 & $43 \pm 21$ & \multirow{4}{*}{17} & 2100 & $0,00-1,95$ & \multirow{4}{*}{1,50} \\
\hline 1 & 1960 & 4,25 & 83 & $45 \pm 25$ & & 1300 & $1,95-2,95$ & \\
\hline 2 & 1490 & 3,08 & 70 & $43 \pm 23$ & & 2200 & $2,95-3,78$ & \\
\hline 2 & 1490 & 4,42 & 81 & $47+19$ & & 1900 & $3,78-4,50$ & \\
\hline 3 & 1100 & 3,50 & 77 & $129 \pm 108$ & \multirow{4}{*}{18} & \multirow{4}{*}{1300} & $0,00-1,42$ & 1,10 \\
\hline 3 & 1100 & 4,58 & 80 & $173 \pm 158$ & & & $1,42-1,75$ & 1,40 \\
\hline 4 & 850 & 3,42 & 76 & $146 \pm 23$ & & & $1,75-2,25$ & 1,70 \\
\hline 4 & 850 & 4,33 & 81 & $111 \pm 85$ & & & $2,25-4,67$ & 2,00 \\
\hline 5 & 514 & 3,42 & 76 & $134 \pm 22$ & \multirow{4}{*}{19} & 500 & $0,00-1,10$ & \multirow{4}{*}{$\begin{array}{l}1,00(\mathrm{t}<3,25 \mathrm{~h}) \\
2,00(\mathrm{t}>3,25 \mathrm{~h})\end{array}$} \\
\hline 5 & 514 & 4,33 & 81 & $139 \pm 82$ & & 650 & $1,10-1,83$ & \\
\hline 6 & 405 & 3,00 & 69 & - & & 1100 & $1,83-5,00$ & \\
\hline 6 & 405 & 4,17 & 81 & $171 \pm 73$ & & - & - & \\
\hline
\end{tabular}

Estireno:água $=0,32: 1,00-[\mathrm{BPO}]=37,40 \mathrm{~g} / \mathrm{L}-\mathrm{T}=84^{\circ} \mathrm{C}-*[\mathrm{PES}]=1,5 \mathrm{~g} / \mathrm{L}$ 
(A)

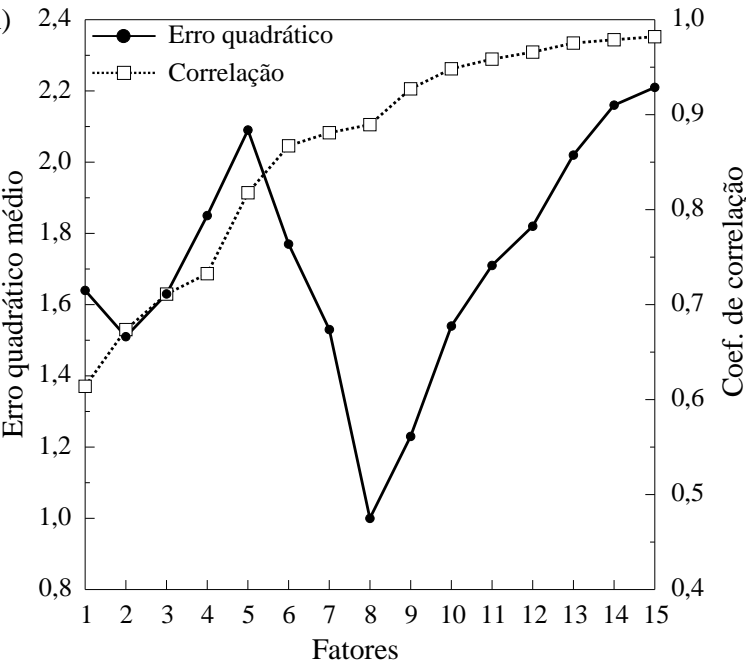

(B)

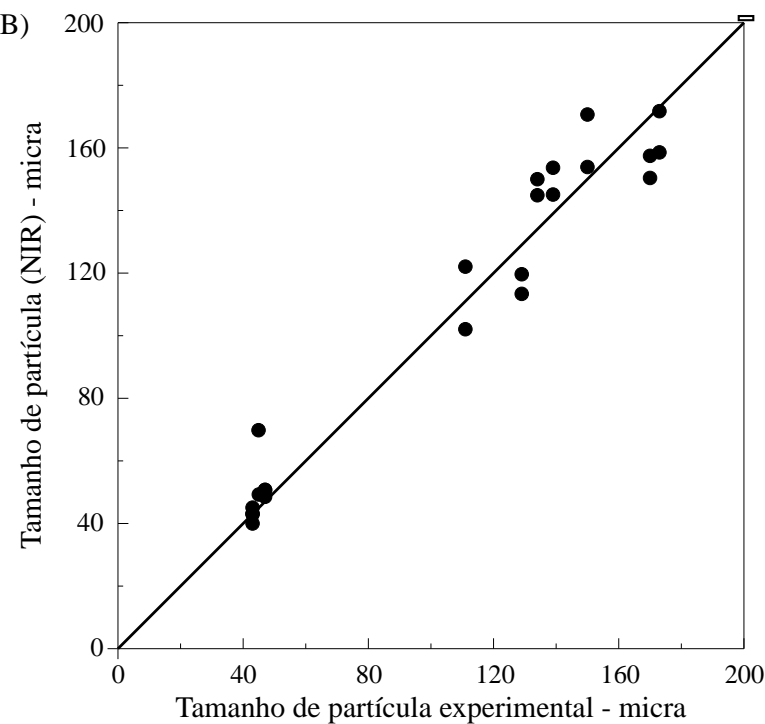

Figura 2. (A) Teste de validação cruzada de modelos PLS e (B) predições do modelo NIRMOD1.

entes de correlação (usando conjunto de dados de calibração). Observa-se que um mínimo de 8 fatores deve ser utilizado para a predição adequada de tamanho médio de partícula a partir de espectros na região NIR. $\mathrm{O}$ modelo a 8 fatores foi denominado NIRMOD1. Na Figura 2B, verifica-se que a capacidade preditiva do modelo para o novo conjunto de dados é muito similar à capacidade do modelo descrito anteriormente (Santos et al., 1998).

O modelo NIRMOD1 foi aplicado no monitoramento do experimento 18 , no qual condições mais complexas de operação foram empregadas para geração de dados para a calibração. Neste experimento, a concentração de agente de suspensão foi incrementada gradativamente ao longo da
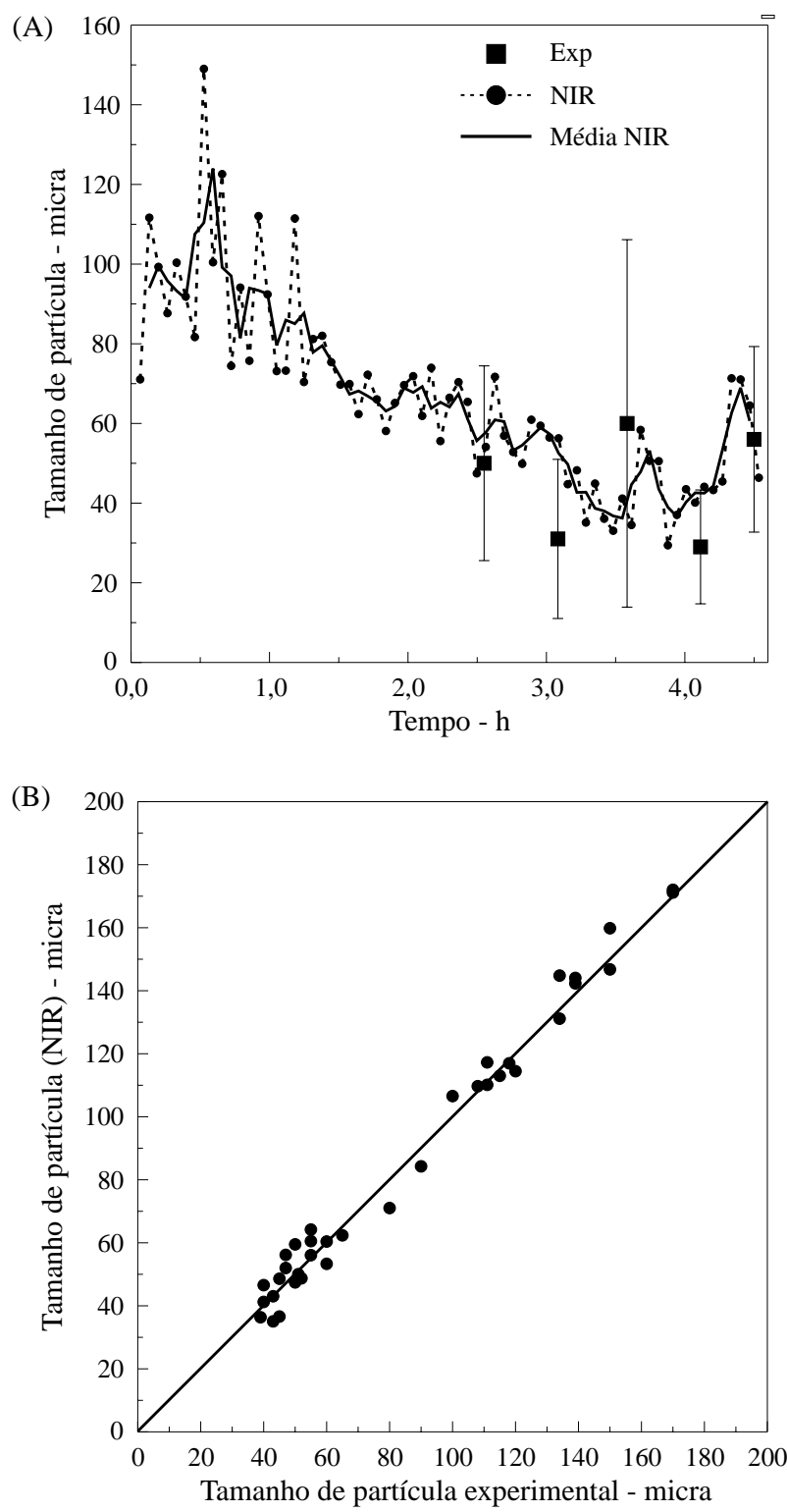

Figura 3. (A) Monitoramento do tamanho de partícula no exp. 18 via espectrofotometria e, (B) predições do modelo NIRMOD2.

batelada. Conforme apresentado na Figura 3A, o modelo de calibração é capaz de acompanhar a tendência dos tamanhos de partícula experimentais obtidos através de microscopia eletrônica (representados por pontos centrados em barras de erro que correspondem a duas vezes o desvio padrão da distribuição), fornecendo informações sobre a dinâmica das gotas/partículas ao longo da reação.

O sucesso no monitoramento do tamanho de partícula no experimento 18 motivou a realização de uma nova estratégia de calibração. Já que o espectrofotômetro realizava predições acuradas de tamanho ao longo do processo, resolveu-se inves- 
tigar a possibilidade de se incluir tais predições do modelo NIRMOD1 no conjunto de dados de calibração, dentro de um contexto de "geração de dados aumentados". Este expediente envolvendo o uso de dados experimentais e predições de modelo vem sendo utilizado com sucesso em trabalhos diversificados (Henriques et al., 1998), possibilitando a obtenção de conjuntos de dados mais uniformes que, se fossem obtidos experimentalmente, demandariam um enorme esforço e um custo proibitivo do ponto de vista do laboratório.

Os resultados da Figura 3A estão uniformente distribuídos na faixa de 30 a $120 \mu \mathrm{m}$. Assim, um novo modelo PLS foi elaborado a partir do conjunto de dados aumentados. Os resultados da calibração a partir do modelo PLS a 8 fatores são apresentados na Figura 3B. Observa-se que nesta abordagem os valores preditos apresentam melhora significativa. Este novo modelo foi denominado NIRMOD2 e utilizado no acompanhamento em linha de tamanho de partículas nos experimentos.

\section{Resultados e discussões}

Conforme exposto na Tabela 1, os experimentos 17,18 e 19 receberam perturbações específicas na velocidade de agitação e concentração de agente de suspensão. Para os propósitos deste trabalho, o mais importante é observar que tais perturbações são efetivas nas alterações da DTP, sem introduzir muitas modificações nas demais variáveis do processo de polimerização. Este aspecto pode ser verificado na Figura 4, na qual se observa semelhança das curvas de conversão e constância dos pesos moleculares ao longo dos experimentos.

As Figuras 5A e 5B apresentam a evolução dos tamanhos médios de partícula para os experimentos 17 e 19 medidos por espectrofotometria e microscopia eletrônica. Observa-se no experimento 17 elevada região de confiança da média experimental da carga final, fruto da bimodalidade da DTP resultante. Tanto no exp. 17 quanto no exp. 19 a tendência dos tamanhos observada pelo espectrofotômetro são confirmadas pelos resultados experimentais, e estão de acordo com a descrição de Kiparissides (1996). Segundo o autor, ocorre inicialmente no reator de polimerização em suspensão um período de redução do tamanho
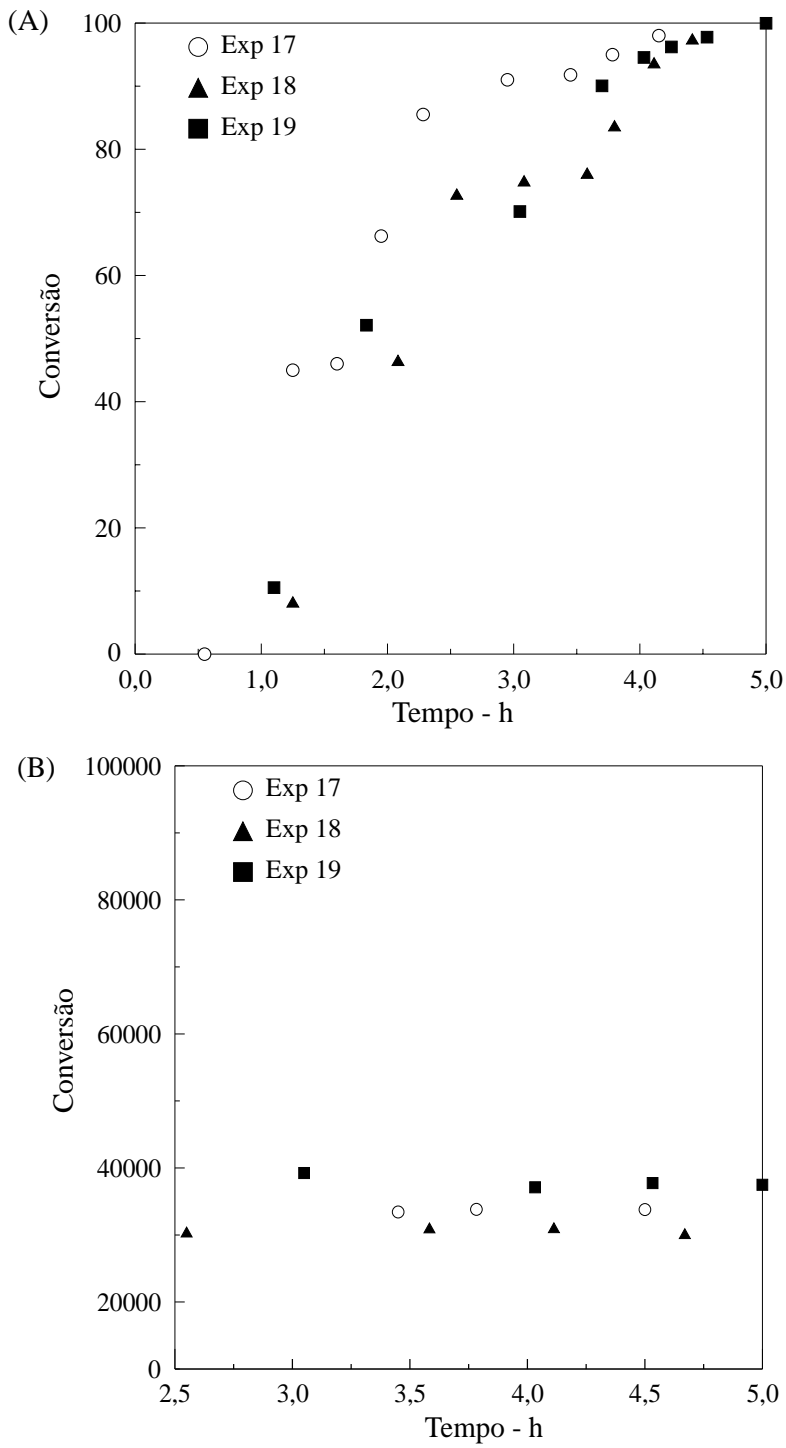

Figura 4. (A) Evolução da conversão e (B) peso molecular ponderal médio para as corridas 17,18 e 19 .

(DTP governada pelos fenômenos de rompimento). Em seguida, um estágio viscoso é estabelecido com correspondente queda da taxa de rompimento (DTP governada pelos fenômenos de coalescência).

Objetivando a obtenção de tamanhos de partícula específicos, foram realizados experimentos com os agentes de suspensão PES (exp. 20) e PVA (exps 21, 22 e 23), utilizando o modelo NIRMOD2 e a estratégia de controle descrita em Santos et al. (1999), a qual estabelece a manipulação da velocidade de agitação no reator de acordo com os tamanhos de partícula preditos pelo espectrofotômetro.

No experimento 20, ajustou-se a concentração de PES em 1,10 g/L e executou-se a reação com 

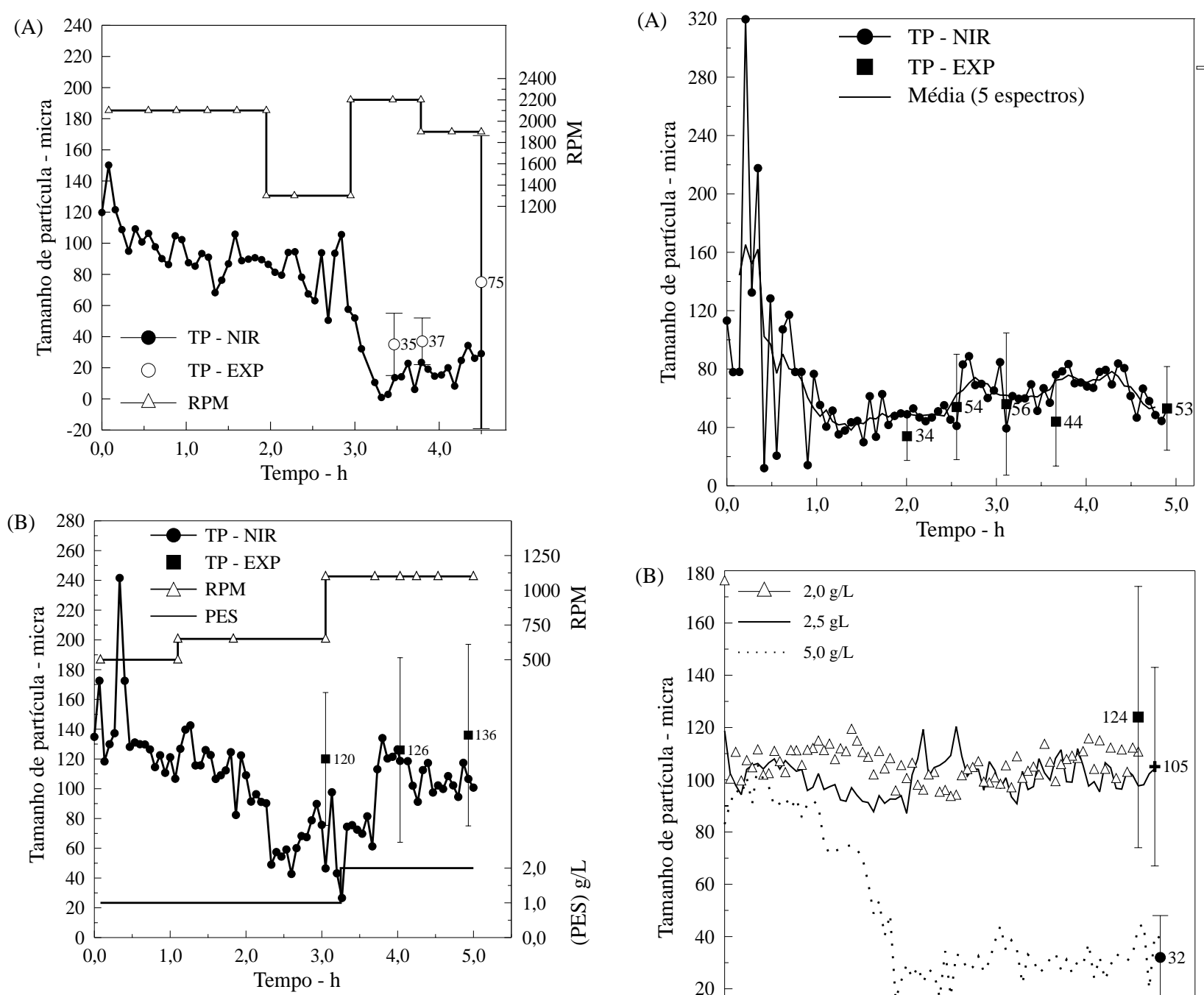

Figura 5. Tamanhos de partícula e condições operacionais: (A) exp. 17 - (B) exp 19.

manipulação da velocidade de agitação, objetivando alcançar o tamanho médio de 60 micra. A Figura 6A mostra que a evolução dos tamanhos preditos pelo espectrofotômetro está em ótima concordância com os dados experimentais e o valor final obtido - 53 micra - está muito próximo do desejado.

Foram realizados dois experimentos utilizando PVA, nas concentrações de 2,0 e $2,5 \mathrm{~g} / \mathrm{L}$, fixando o valor desejado de tamanho de partícula em 110 micra (exps 21 e 22). Um terceiro experimento (exp.23) foi realizado com PVA a $5,0 \mathrm{~g} / \mathrm{L}$, visando alcançar o tamanho de 30 micra. A Figura $6 \mathrm{~B}$ apresenta os resultados obtidos nos experimentos com PVA, indicando a elevada concordância entre os valores de tamanho de partícula preditos pelo espectrofotômetro e o os dados experimentais, bem como a alta capacitação

(B)

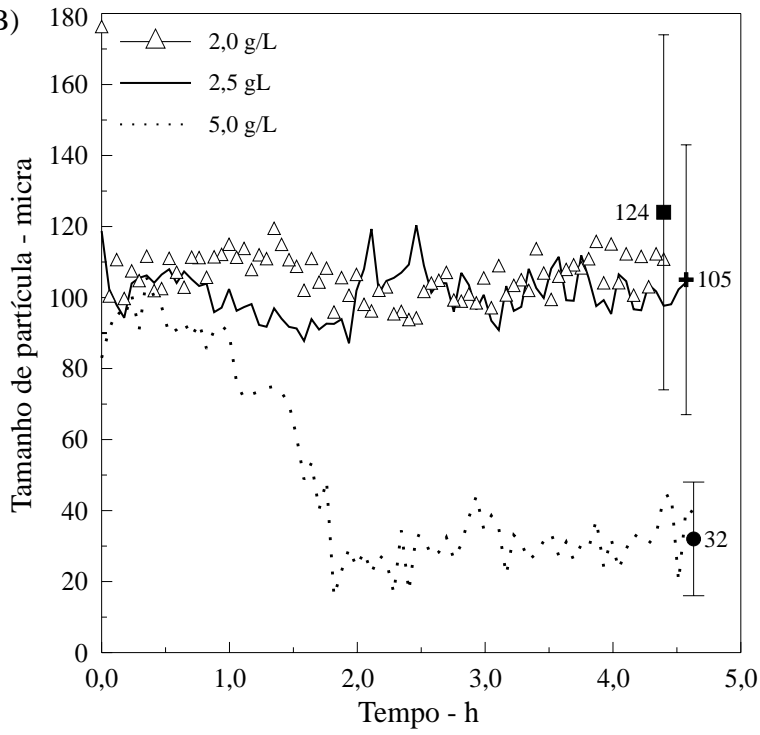

Figura 6. Acompanhamento do tamanho de partícula: (A) exp 20 e (B) exps 21, 22 e 23.

do instrumento para prover informações sobre a dinâmica das partículas.

\section{Conclusão}

Este trabalho demonstra que, o uso combinado da espectrofotometria de infravermelho próximo e a técnica de regressão nos mínimos quadrados parciais (PLS) permite a determinação em tempo real de tamanhos de partícula em reatores de polimerização de estireno em suspensão. Os resultados demonstram que os espectros de absorção são capazes de responder a diferentes condições operacionais, fornececendo informações sobre a dinâmica das par- 
tículas. O uso da técnica na obtenção de resinas com tamanho de partícula especificado foi bem sucedido, mesmo com a substiutição do agente de suspensão utilizado na calibração (PES) por um outro (PVA). Conclui-se que a espectrofotometria NIR pode ser efetivamente aplicada no controle em linha de tamanhos médios de partícula na polimerização do estireno em suspensão.

\section{Referências Bibliográficas}

1. Bishop, R.B., Pratical Polymerization for Polystyrene, Cahners publishing Co., Inc (1971).

2. Bjorsvik, H., Bjorsvik, H., "Reaction Monitoring in Explorative Organic Synthesis Using FiberOptical NIR Spectroscopy and Principal Component Analysis", Applied Spectroscopy., 50, pp. 1541 - 1544 (1996).

3. Chatzi, E.G., Boutris, C.J., Kiparissides, C., “OnLine Monitoring of Drop Size Distributions in Agitaded Vessels. 1. Effects of Temperature and Impeller Speed", Ind. Eng. Chem. Res., 30, pp. 536 - 543 (1991).

4. Clifford, R.H., Tan, H.M., Liu, H.Y., et al., "Particle Size measurements in the submicron range by the differential electromobility technique: comparison of aerosols from thermospray, ultrasonic, pneumatic and frit-type nebulizers", Spectrochimica Acta part B-atomic Spectroscopy, 48, pp. 1221 - 1235 (1993).

5. Co, C.C., Kaler, E.W., "Particle Size and Monomer Partioning in Microemulsion Polymerization. 2 - Online Small Angle Neutron Scattering Studies", Macromolecules, 31, pp. 3203 - 3210 (1998).

6. Grulke, A.E., "Suspension Polymerization", In: Encyclopedia of Polymer Science and Engineering, v. 16, New York, Wiley \& Sons, pp. 443-472 (1989).

7. Hall, J.W., Mcneill, B., Rollins, M.J., Draper, I., Thompson, B.B. e Macaloney, G., "NearInfrared Spectroscopic Determination of Acetate, Ammonium, Biomass, and Glycerol in an Industrial Escherichia coli Fermentation", Applied Spectroscopy.,50, pp. $102-108$ (1996).
8. Henriques, A.W.S., Costa, A.C., Lima, E.L. e Alves. T.L.M., In: $20^{\text {th }}$ Symposium on Biotechnology for Fuels and Chemicals, 81, Gatlinburg, USA, (1998).

9. Kiparissides, C., "Polymerization Reactor Modeling: A Review of Recent Developments and Future Directions", Chem. Engng. Sci., 51, pp. 1637 - 1659 (1996).

10. Liu, D., Rutherford, D., Kinsey, M., e Prather, K.A., "Real-time Monitoring of Pyrotechnically Derived Aerosol Particles in the Troposphere", Analytical Chemistry,69, pp. $1808-1814$ (1997).

11. NSAS 3.0, REFERENCE MANUAL FOR NEAR INFRARED SPECTRAL ANALYSIS, NIRSystems Inc, EUA (1993).

12. Popp, J., Lankers, M., Trunk, M., Hartmann, I., Urlaub, E. e Kiefer W., "High-Precision Determination of Size, Refractive Index, and Dispersion of Single Microparticles from Morphology-Dependent Resonances in Optical Processes.", Applied Spectroscopy, 52, 2, pp. $284-291$ (1998).

13. Rubin, I I., "Injection Molding, Theory \& Practice", In: SPE monographs, v.1, EUA, John Wiley \& Sons, Inc (1972).

14. Santos, A. F., Lima, E. L. e Pinto, J. C., "In-line Evaluation of Average Particle Size in Styrene Suspension Polymerizations Using NearInfrared Spectroscopy", Journal of Applied Polymer Science, 70, 1737 - 1745 (1998).

15. Santos, A. F., Lima, E. L. E Pinto, J. C., "Control and Design of Average Particle Size in Styrene Suspension Polymerizations Using NIRS", Journal of Applied Polymer Science (submetido) (1999).

16 Sarvetnick, H. A., Pol yvinyl Chloride - Plastics Applications Series, Nova Iorque - EUA, Reinhold Book Co (1969).

17. Siesler, H.W.,"Near -Infrared Spectroscopy of Polymers", Makromol. Chem., Macromol. Symp., 52, pp. 113 - 129 (1991).

18. Yuan, H. G., Kalfas, G., Ray, W. H., "Suspension Polymerization", JMS-Rev. Macromol. Chem. Phys., C31, (2\&3), pp. 215 - 299 (1991). 